\title{
Numerical investigation of the effect of number of zones on Fresnel Zone Plates imaging performance under different coherence conditions
}

\section{Ying Li, Ombeline de la Rochefoucauld, Philippe Zeitoun}

Ying Li, Ombeline de la Rochefoucauld, Philippe Zeitoun, "Numerical investigation of the effect of number of zones on Fresnel Zone Plates imaging performance under different coherence conditions," Proc. SPIE 11886, International Conference on X-Ray Lasers 2020, 118860K (8 July 2021); doi: $10.1117 / 12.2591971$ 


\title{
Numerical Investigation of the Effect of Number of Zones On Fresnel Zone Plates Imaging Performance Under Different Coherence Conditions
}

\author{
Ying Li ${ }^{\mathrm{a}, \mathrm{b}}$, Ombeline De La Rochefoucauld ${ }^{\mathrm{b}}$, and Philippe Zeitoun ${ }^{\mathrm{a}}$ \\ aLaboratoire d'Optique Appliquée, CNRS, ENSTA-Paris, Institut Polytechnique de Paris, 828 \\ Boulevard des Maréchaux, 91120 Palaiseau, France \\ b Imagine Optic, rue François Mitterrand, 33400 Talence, France
}

\begin{abstract}
In recent years, integral imaging has received increasing attention as a promising 3D imaging technique with one single exposure. In medical diagnostics, the X-ray integral imaging system potentially has a much shorter exposure time than the conventional computed tomography, reducing the radiation damage to the patient. By replacing the micro-lens array with a micro-Fresnel Zone Plate (FZP) array, the classical visible integral imaging system can be transposed to an X-ray system. However, limited by the micro-scale dimensions of FZP in the array and current manufacturing techniques, the number of zones of FZP is required to be small. This may have an important impact on the FZP imaging performance. Based on the scalar diffraction theory, this paper introduces a simulation method and numerically investigated the effect of the number of zones on the FZP imaging performance under different coherence conditions.
\end{abstract}

Keywords: Fresnel zone plate; modeling; wave propagator; coherence; X-ray integral imaging

\section{INTRODUCTION}

Nowadays, computed tomography (CT) is one of the most common 3D imaging techniques in clinical diagnosis, providing highly accurate information of internal structure and guiding medical treatment. The principle of $\mathrm{CT}$ is rendering 3D image of the sample from multiple projections recorded under different angles of view. It is apparent that large projection number will bring a better reconstruction quality. ${ }^{1}$ Unfortunately, it will meanwhile deliver a high radiation dose for the patients and increase the probability of cancer. ${ }^{2,3} \mathrm{X}$-ray integral imaging can provide an alternative to $\mathrm{CT}$ in medical imaging as it has the prospect of reconstructing 3D images from one single capture, consequently leading to a lower dose and a shorter exposure time.

Integral imaging approach, first proposed by Lippmann in $1908,{ }^{4}$ consists in capturing simultaneously the spatial and angular information of the light ray intersecting on the photosensor, the so-called 4D light field. The captured 4D light field allows us to refocus the image on different planes and render a 3D model of the sample with one exposure. ${ }^{5,6}$ For visible light, various integral imaging acquisition systems have been proposed, such as camera arrays ${ }^{7}$ micro-lens arrays ${ }^{8}$ and amplitude masks. ${ }^{9}$ The design of placing a micro-lens array in the front of conventional camera's photosensor is the most widespread, in view of the diversity and availability of micro-lens array. However, to convert the integral imaging system from visible light to X-ray, we must search alternative optical components for the refractive lenses. Because the refractive index is very close to unity for all materials at X-ray wavelengths, leading to a extremely long focal distances at kilometer scale for a single lens and a low numerical aperture. ${ }^{10}$

The Fresnel Zone Plate (FZP), relying on the diffraction to realize X-ray focusing, is widely used and has been demonstrated to achieve a high spatial resolution. ${ }^{11}$ The FZP array is thus an available alternative to the refractive lens array in X-ray imaging. ${ }^{12-14}$ FZP geometry dominates its imaging performance. For a desired

Further author information: (Send correspondence to Y.L. and P.Z.)

Y.L.: E-mail: ying.li@ensta-paris.fr

P.Z.: E-mail: philippe.zeitoun@ensta-paris.fr

International Conference on X-Ray Lasers 2020, edited by Davide Bleiner,

Proc. of SPIE Vol. 11886, 118860K · C 2021 SPIE · CCC code:

0277-786X/21/\$21 - doi: $10.1117 / 12.2591971$

Proc. of SPIE Vol. $11886118860 \mathrm{~K}-1$ 
focus, the larger the number of zones is, the higher resolution FZP can attain. Here, the resolution refers to the width of the focused point. Therefore, its number of zones is one of the most important structural parameters. Yet, due to the micro-scale dimensions of FZP in the array of an integral imaging system and current manufacturing techniques, the number of zones of FZP is greatly limited.

Therefore, this paper attempts to numerically investigate the impact of FZP number of zones on its imaging performance under different coherence conditions, especially the case of very small number of zones. Accordingly, a simulation method based the scalar diffraction theory is introduced in this paper. Several simulation results of FZP imaging are presented and discussed, then compared to ideal refractive lens.

\section{METHOD}

A Fresnel zone plate is a diffractive optic, its alternating transparent and opaque zones make the incident light constructively interfere at the desired focus. The FZP's focusing properties can be analyzed by the scalar diffraction theory. Figure 1 represents a generalized schematic of a FZP optical system.

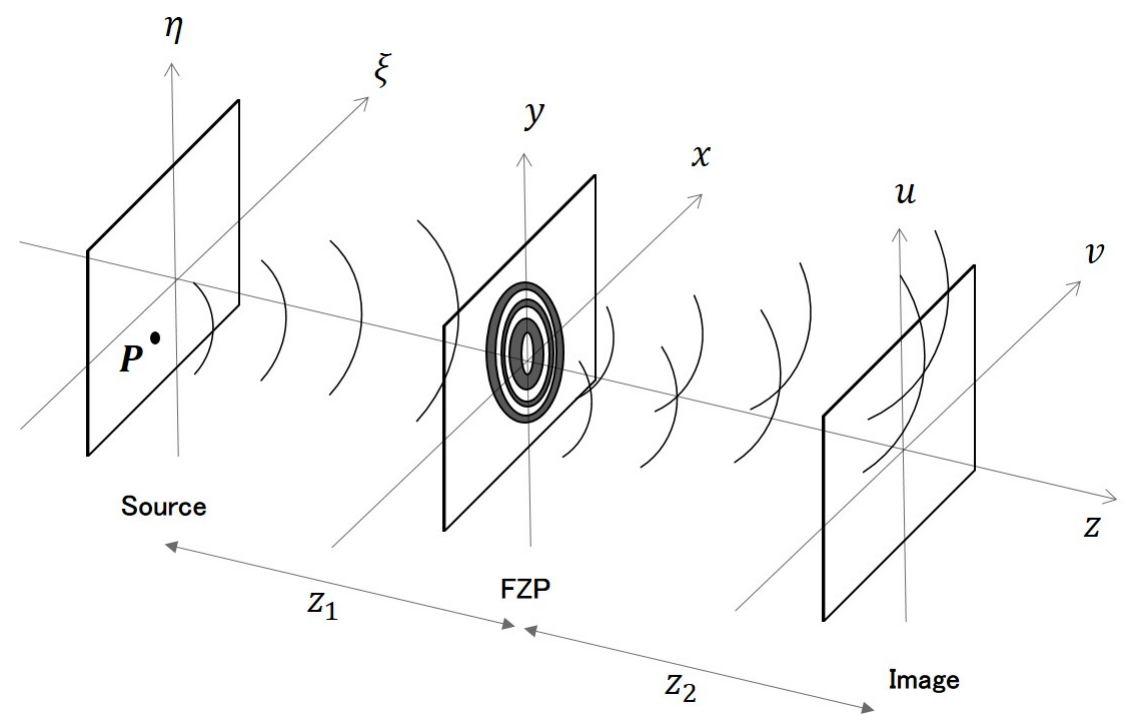

Figure 1. Generalized schematic of Fresnel Zone Plate (FZP) optical system: the point source $\boldsymbol{P}$ on the plane $(\xi, \eta)$ emitting a spherical wave is intercepted and diffracted by the FZP on the plane $(x, y)$. The image of the source is formed on the plane $(u, v) . z_{1}$ and $z_{2}$ represent respectively the source-FZP distance and the FZP-image distance.

Consider a point source $\boldsymbol{P}(\xi, \eta)$ emitting spherical wave diffracted by a FZP on the plan $(x, y)$ and its image formed on the plane $(u, v)$. The distances between the point source to the FZP and the FZP to the image are respectively noted as $z_{1}$ and $z_{2}$. According to Huygens-Fresnel principle and the linearity of the optical system, ${ }^{15}$ the impulse response $h(\xi, \eta, u, v)$ of the FZP can be expressed in the form of a Fourier Transform

$$
h(0,0, u, v)=\frac{z_{2}}{z_{1}} \exp \left[\frac{i k}{2 z_{2}}\left(u^{2}+v^{2}\right)\right] \times \mathcal{F}\left\{t\left(\lambda z_{2} x^{\prime}, \lambda z_{2} y^{\prime}\right) \exp \left\{\frac{i k}{2}\left(\frac{1}{z_{1}}+\frac{1}{z_{2}}\right)\left[\left(\lambda z_{2} x^{\prime}\right)^{2}+\left(\lambda z_{2} y^{\prime}\right)^{2}\right]\right\}\right\}
$$

where $t(x, y)$ is the transmission function of the FZP, with $x^{\prime}=\frac{x}{\lambda z_{2}}, y^{\prime}=\frac{y}{\lambda z_{2}}$.

The image $\operatorname{Im} g(u, v)$ can be obtained by the convolution of the image predicted by geometrical optics $i m g(u, v)$ with the impulse response $h(\xi, \eta, u, v) .{ }^{16}$ It is worth noting that the application of the point spread function (PSF) on an image depends on the degree of coherence of the source. Here, we consider two extreme cases, fully coherent and fully incoherent. The image $\operatorname{Img}(u, v)$ can be written as

$$
\operatorname{Img}(u, v)= \begin{cases}|h(\xi, \eta, u, v) \otimes i m g(u, v)|^{2} & \text { fully coherent } \\ |h(\xi, \eta, u, v)|^{2} \otimes|i m g(u, v)|^{2} & \text { fully incoherent }\end{cases}
$$


According to the convolution theorem, Equation (2) can be further converted to

$$
\operatorname{Img}(u, v)= \begin{cases}\mathcal{F}^{-1}\left\{|\mathcal{F}\{h(\xi, \eta, u, v)\} \times \mathcal{F}\{i m g(u, v)\}|^{2}\right\} & \text { fully coherent } \\ \mathcal{F}^{-1}\left\{\mathcal{F}\left\{|h(\xi, \eta, u, v)|^{2}\right\} \times \mathcal{F}\left\{|i m g(u, v)|^{2}\right\}\right\} & \text { fully incoherent }\end{cases}
$$

This conversion reduces the complexity of the computation and allows greatly increasing the computation speed thanks to the use of FFT.

This method is flexible for the definition of FZP and the adjustment of imaging system structure, allowing to study the effects of different parameters on the FZP imaging performance. More detailed derivation and validation tests about this method are provided in reference. ${ }^{17}$

\section{RESULTS}

To our best knowledge, the quality of the image formed by a FZP versus the number of zones has never been studied. In this section, by the proposed method, the images of the FZP with different $N$ were performed and compared to the images formed by the refractive lens, respectively, under fully incoherent and fully coherent sources. The effect of the number of zones on FZP imaging performance is then discussed.

\subsection{Fully incoherent case}

A series of numerical images of a resized 1951 USAF resolution test chart formed by FZP with increasing $N$ are displayed in Figures 2a-e and the part of interest on the test chart is zoomed in Figure 2f. In the following text, the blue-, orange-, and green-colored bars in Figure $2 \mathrm{f}$ are named as elements 2.2, 2.3, and 2.4, respectively, with the width of $1.36 \mu \mathrm{m}, 1.18 \mu \mathrm{m}$, and $0.99 \mu \mathrm{m}$ on the image plane. Figures $2 \mathrm{a}-\mathrm{e}$ are carried out with a fully incoherent source of $11 \mathrm{keV}$. The object to FZP distance is $3 f$, leading to the creation of an image at $1.5 f$. It is apparent from Figures $2 \mathrm{a}-\mathrm{e}$ that as $N$ increases, the first order image becomes sharper. As the radius of FZP is meanwhile broadened along $N$, the field of view is enlarged.

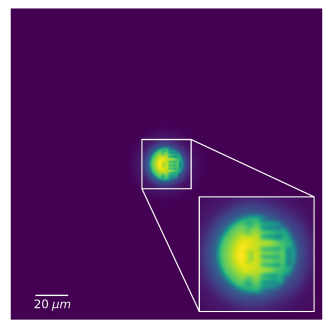

(a) $N=5$

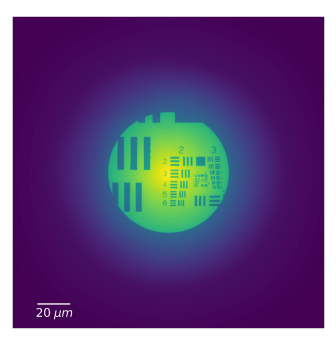

(d) $N=60$

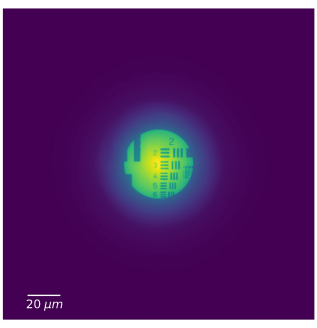

(b) $N=20$

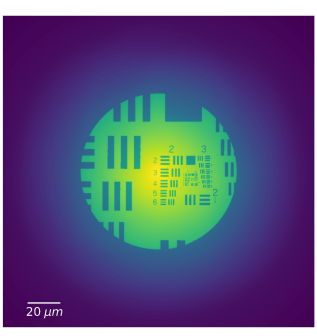

(e) $N=100$

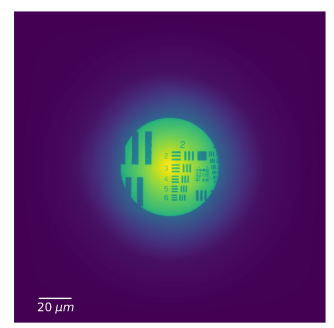

(c) $N=40$

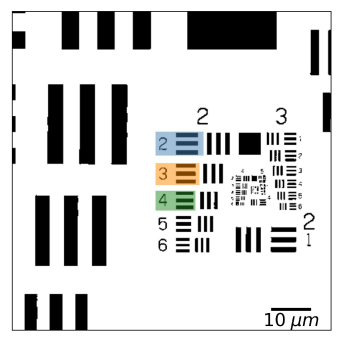

(f) Test chart

Figure 2. (a-e) False color numerical images of the USAF 1951 test target imaged by FZP with different number of zones under fully incoherent illumination. The object-FZP distance is $3 f$ and the FZP-image distance is $1.5 f$. Each image dimension is $200 \times 200 \mu \mathrm{m}^{2}$. (f) Zoomed part of interest on the test chart: for the following part, the blue-, orange- and green-colored elements of bars in group 2 are respectively noted as elements $2.2,2.3$, and 2.4. 
Measured the contrast of Figures $2 \mathrm{a}-\mathrm{e}$, we remark the maximum contrast value is is as low as 0.2 (see Figure 7 in References [17]). In order to study its origin, we modeled the image formation with a theoretical perfect refractive lens. Supposed the FZPs in Figure 2 can be replaced by the refractive lenses with the same numerical aperture (NA), the transmission of refractive lens $t_{\text {Lens }}(x, y)$ is defined as

$$
t_{\text {lens }}(x, y)=\left\{\begin{array}{l}
\exp \left[-\frac{i k}{2 f}\left(x^{2}+y^{2}\right)\right], \sqrt{x^{2}+y^{2}}<R \\
0, \text { else }
\end{array}\right.
$$

where $R$ and $f$ are the radius and focal length of lens, respectively.

A group of images formed by the refractive lens is simulated under the same structure of Figure 2, displayed in Figure 3. For the ease of comparison with Figure 2, the NA of refractive lens is written as a function of the number of zones. Compared with Figure 2, the intensity of the refractive lens images is obviously more uniform, and there is no longer a halo around the image. The contrast value measured from refractive lens images can reach a maximum of 0.94, much higher than that of FZP images (see Figure 9 in References [17]). This is because the first order image of FZP overlaps with the other orders, resulting in a brightened and complex background.

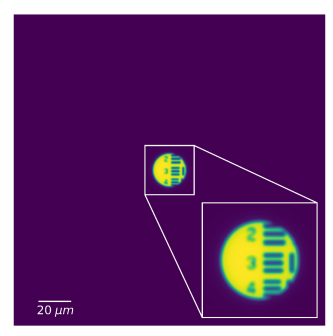

$N A(N=5)$

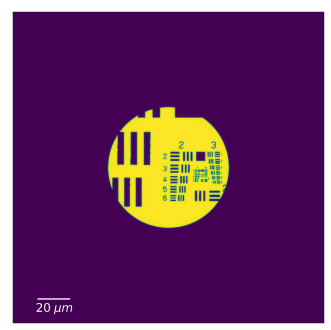

$N A(N=60)$

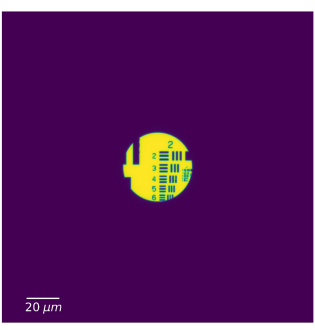

$N A(N=20)$

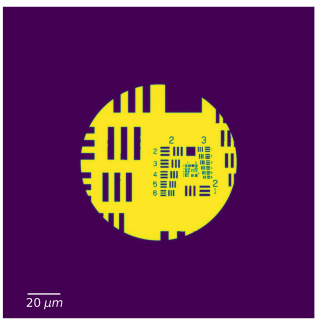

$N A(N=100)$

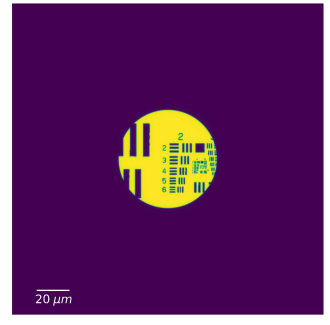

$N A(N=40)$

Figure 3. False color numerical images of the USAF 1951 test target imaged by refractive lens under fully incoherent illumination. The NA of the refractive lens equals to the NA of the FZP when the number of zones of FZP is 5, 20, 40, 60, and 100. Each image dimension is $200 \times 200 \mu \mathrm{m}^{2}$.

\subsection{Fully coherent case}

Keeping the same configuration as mentioned in the fully incoherent case, we discuss FZP imaging performance under fully coherent illumination in this section. The simulated images of FZP versus N are shown in Figure 4. As $\mathrm{N}$ increases, the first order image of FZP becomes more resolved, while the images of the other orders are much more apparent in the fully coherent case than in the fully incoherent images. To visualize more directly the FZP images difference of images under different coherence conditions, Figure 5 displays side by side the fully incoherent and fully coherent FZP images for $N=100$. Unlike the other orders' images with recognizable details in the fully coherent case, we can only see a diffuse halo overlapping the first order image in the fully incoherent case. Indeed, the overlap of different order images substantially degrades the first order image quality compared to the fully incoherent case. 


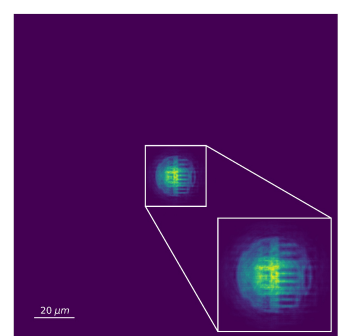

$N=6$

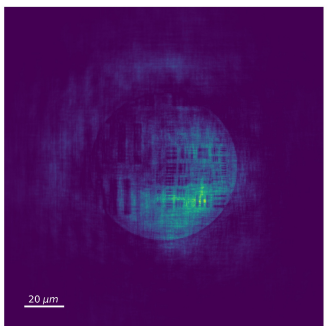

$N=60$

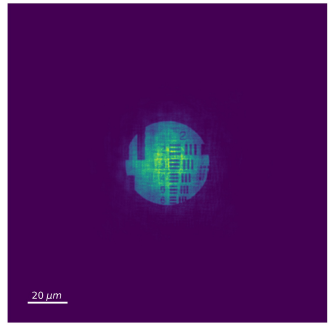

$N=20$

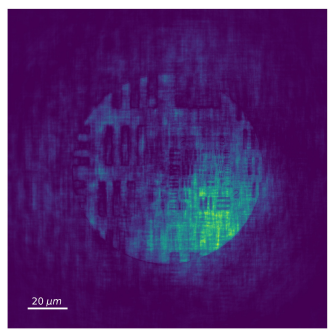

$N=100$

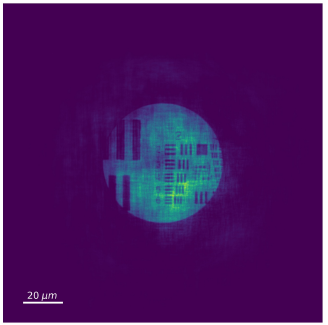

$N=40$

Figure 4. False color numerical images of the USAF 1951 test target imaged by FZP with different number of zones under fully coherent illumination. The object-FZP distance is $3 f$ and the FZP-image distance is $1.5 f$, as same as for Figure 2 . Each image dimension is $160 \times 160 \mu \mathrm{m}^{2}$.

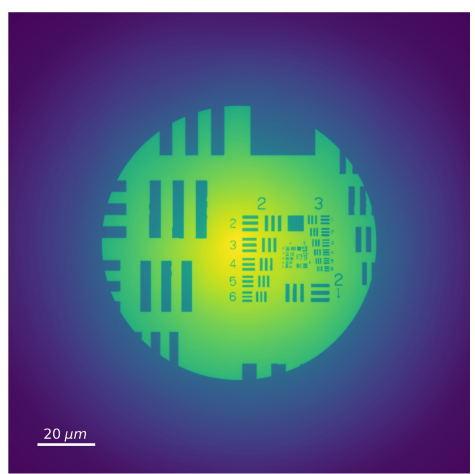

(a) Fully incoherent

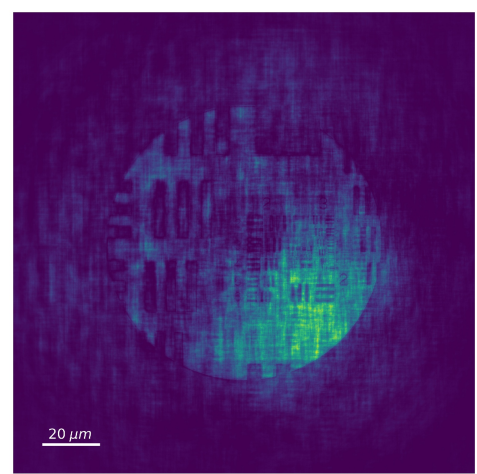

(b) Fully coherent

Figure 5. Comparison between fully incoherent and fully coherent images formed by FZP. The other orders images become more visible in the fully coherent case than the fully incoherent case, greatly degrading the first order image quality. $N$ is equal to 100 . Each image dimension is $160 \times 160 \mu \mathrm{m}^{2}$.

To bring out the characteristics of a FZP image, we can compare the Figure 4 to Figure 6 . The set of images in Figure 6 are simulated with the refractive lenses of the same NA as the FZPs in Figure 4 . Without the influence of different orders images, the refractive lens images are clearer and more visible than FZP ones. On the other hand, we can see the diffraction patterns of each element of the test chart appear under fully coherent illumination in Figure 6, which shows the impact of coherence in imaging. On closer observation, we can also find the diffraction pattern changes with different $N$, which is related to the divergence of the source in our model. The images can be regarded as a result of collective interference between each point of the test chart. The variation of the diffraction pattern then leads to the oscillation of the image intensity along $N$. In the fully coherent case, strong interference results in more complex images. It is complicated to quantify the evolution of the image by the contrast as used in the fully incoherent case. 


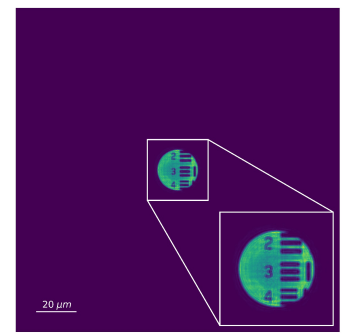

$N A(N=6)$

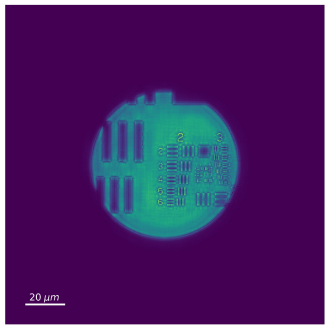

$N A(N=60)$

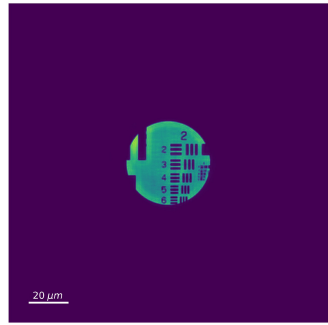

$N A(N=20)$

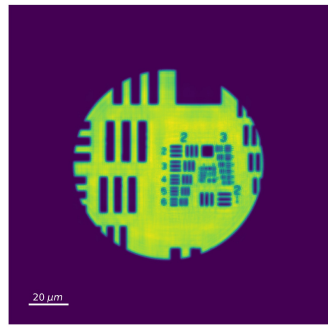

$N A(N=100)$

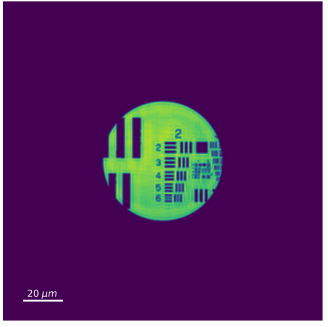

$N A(N=40)$

Figure 6. False color numerical images of the USAF 1951 test target imaged by refractive lens with different number of zones under fully coherent illumination. The NA of the refractive lens equals to the NA of the FZP in Figure 4 when the number of zones of FZP is 5, 20, 40, 60, and 100. Each image dimension is $160 \times 160 \mu \mathrm{m}^{2}$.

\section{CONCLUSIONS}

In this paper, a simulation method based on scalar diffraction has been introduced. The presented method is able to simulate the images formed by FZP with different parameters and under different coherence conditions. The effect of the number of zones on image formation has been discussed under fully incoherent and fully coherent conditions, especially the case of very small number of zones. The results have shown that surprisingly image can be formed with a number of zones as low as 5. However, due to the overlap of different orders of FZP, the images have relatively low contrast, and this influence of the other orders becomes more important in the fully coherent case than in the fully incoherent case. The reported results in this paper bring helpful information for the design of FZP for an integral imaging system in X-ray. Future study can be extended to the axial effect of the number of zones, the formation of images in the case of partially coherent beam and the application on the full set-up of integral imaging system.

\section{ACKNOWLEDGMENTS}

This work has received funding from the European Union's Horizon 2020 research and innovation program under grant agreement no 871124 Laserlab-Europe,and Prematuration 2019 project from IP Paris.

\section{REFERENCES}

[1] Van Daatselaar, A., Van der Stelt, P., and Weenen, J., "Effect of number of projections on image quality of local ct," Dentomaxillofacial Radiology 33(6), 361-369 (2004).

[2] De González, A. B., Mahesh, M., Kim, K.-P., Bhargavan, M., Lewis, R., Mettler, F., and Land, C., "Projected cancer risks from computed tomographic scans performed in the united states in 2007," Archives of internal medicine 169(22), 2071-2077 (2009).

[3] Mathews, J. D., Forsythe, A. V., Brady, Z., Butler, M. W., Goergen, S. K., Byrnes, G. B., Giles, G. G., Wallace, A. B., Anderson, P. R., Guiver, T. A., et al., "Cancer risk in 680000 people exposed to computed tomography scans in childhood or adolescence: data linkage study of 11 million australians," Bmj 346 (2013).

[4] Lippmann, G., "Epreuves reversibles donnant la sensation du relief," (1908). 
[5] Ng, R., Levoy, M., Brédif, M., Duval, G., Horowitz, M., and Hanrahan, P., Light field photography with a hand-held plenoptic camera, PhD thesis, Stanford University (2005).

[6] Tian, L. and Waller, L., "3d intensity and phase imaging from light field measurements in an led array microscope," optica 2(2), 104-111 (2015).

[7] Wilburn, B., Joshi, N., Vaish, V., Talvala, E.-V., Antunez, E., Barth, A., Adams, A., Horowitz, M., and Levoy, M., "High performance imaging using large camera arrays," in [ACM SIGGRAPH 2005 Papers], 765-776 (2005).

[8] Georgiev, T. G., Zheng, K. C., Curless, B., Salesin, D., Nayar, S. K., and Intwala, C., "Spatio-angular resolution tradeoffs in integral photography.," Rendering Techniques 2006(263-272), 21 (2006).

[9] Veeraraghavan, A., Raskar, R., Agrawal, A., Mohan, A., and Tumblin, J., "Dappled photography: Mask enhanced cameras for heterodyned light fields and coded aperture refocusing," ACM Trans. Graph. 26(3), 69 (2007).

[10] Attwood, D. and Sakdinawat, A., [X-rays and extreme ultraviolet radiation: principles and applications], Cambridge university press (2017).

[11] Erko, A., Idir, M., Krist, T., and Michette, A. G., [Modern developments in X-ray and neutron optics], vol. 137, Springer (2008).

[12] Le Pape, S., Zeitoun, P., Idir, M., Dhez, P., Ros, D., Carillon, A., Roca, J., and François, M., "Wavefront measurements in the soft x-ray range," The European Physical Journal Applied Physics 20(3), 197-203 (2002).

[13] Le Pape, S., Zeitoun, P., Idir, M., Dhez, P., Rocca, J., and François, M., "Electromagnetic-field distribution measurements in the soft x-ray range: full characterization of a soft x-ray laser beam," Physical review letters 88(18), 183901 (2002).

[14] Zeitoun, P., Balcou, P., Bucourt, S., Delmotte, F., Dovillaire, G., Douillet, D., Dunn, J., Faivre, G., Fajardo, M., Goldberg, K., et al., "Recent developments in x-uv optics and x-uv diagnostics," Applied Physics B 78(7-8), 983-988 (2004).

[15] Gaskill, J., [Linear Systems, Fourier Transforms, and Optics], vol. 576, Wiley (1978).

[16] Goodman, J. W., [Introduction to Fourier optics], Roberts and Company Publishers (2005).

[17] Li, Y., De La Rochefoucauld, O., and Zeitoun, P., "Simulation of fresnel zone plate imaging performance with number of zones," Sensors 20(22), 6649 (2020). 